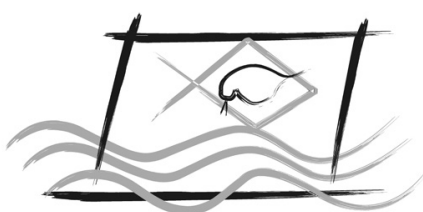

ECOTOX - BRASIL

\title{
Balance disturbance of aquatic microbial microcosm as a method to evaluate the contamination by toxic substances
}

\author{
RIBEIRO, I.A. \\ Departamento de Saúde Ambiental - Faculdade de Saúde Pública - Universidade de São Paulo, São Paulo - SP, Brasil
}

(Received March 04, 2016; Accept March 01, 2017)

\begin{abstract}
Toxicity tests are useful to assess relative toxicities of chemicals in the aquatic environment. Although, they have the advantages of standardized protocols, the test organisms are isolated from the context of the natural conditions. This experimental research challenges the development of an aquatic microbial microcosm with an ecological structure, to evaluate the effects of contamination by toxic substances in the aquatic environment. Two morphological groups were observed in typical ecological succession, according to the modification of the medium. The laboratorial set-up consisted of keeping an organic substrate of $350 \mathrm{~m} \mathrm{~L} \mathrm{~L}^{-1}$ of BOD, placed in glass containers maintained in continuous aeration. Growth of filamentous bacteria was observed after $24 \mathrm{~h}$, followed by significant exponential growth of free swimming ciliates protozoa, reaching its maximum rate on the $4^{\text {th }}$ day. Disturbances were caused by the introduction of copper sulphate and insecticide dichlorvos. Complete inhibitory effect of growth was observed to ciliates at concentrations of $12.28 \mathrm{mg} \mathrm{L}^{-1} \mathrm{Cu}$ and $500 \mathrm{mg} \mathrm{L}^{-1}$ DDVP-20. Easy to be assembled, the method suggested information about theoretical aspects of self-organization. To greater precision, we mention the study of ecological interactions, opened system development, biodiversity molecular approach and application of ecological models.
\end{abstract}

Keywords: aquatic microbial microcosm, evaluation, microbial succession, model, theoretical ecology, toxicity

\section{INTRODUCTION}

As water resources diminish, the need to understand the effects that contaminants may have on aquatic organisms and ecosystems increases in importance (Nikinmaa, 2014). Many efforts have been employed to develop toxicity tests to predict and evaluate both ecological and health effects caused by a wide variety of synthetic chemicals and organic waste potentially toxic from industry and agriculture that are discharged directly or indirectly into the aquatic environment. The control and evaluation of aquatic pollution has been made by permissible limits of toxic substances and bioassays.

About the control by known substances, the main limitation is that it is impossible for determining the biological susceptibility caused by all the toxics which compose a mixture (Rand, 1995). Moreover, many chemicals that enter the environment do not remain in their original form (Wang et al., 2011) or yet, harmful effects of contaminants on the ecosystems and humans cannot be assessed by standard chemical analysis (Logar \& Vodovnik, 2007).
Therefore, early detection of toxic compounds and their biological effects on organisms has become increasingly important (Logar \& Vodovnik, 2007). It is worth mentioning the development of bioassays, used as a preliminary assessment of the presence of biological inhibitors in the aquatic environment (Fiskejö, 1993); (Ribeiro, 1997, 1999).

Although the bioassays can more securely evaluate the impact of an effluent in the water, the test organisms in general, are isolated to their environment and the dynamic context of the natural conditions. Furthermore, Branco (1986) pointed out that the efforts of sanitarians are directed to develop synthetic methods - i.e., the nature of synthesis combining separate elements to form coherent whole (Thorpe, 2010).

According to Ghiglione et al. (2016), the originality of microbial ecotoxicology lies in the role of microorganisms in the ecodynamic of the pollutants which is not strictly taken into account in ecotoxicology. To Colwell (2012), the microbial aspects of ecotoxicology should be explored for discovery and application in environmental pollution. In 
Brandt et.al. (2004), the experiments with natural assemblages of microorganisms should provide an attractive alternative to single-species microbial toxicity tests.

Microcosm is referred as an experimental small simplified ecosystem (Odum \& Barrett, 2014) and began to be used to study the ways in which the aquatic ecosystems are affected and respond to the disruptions caused by chemicals (Cairns $\&$ Niederlehner, 1995). Despite the difficulty of quantitative study of the effects of toxicants in aquatic ecosystems, in particular, due to the consequence of self-organization mechanism (Beyers \& Odum, 1993), the development of experimental models of smaller scale, for microbial community structure, function and dynamics aims to define protocols (Wider et al., 2016).

To Curtis (2007), microbial ecology is going to become 'big science'. Widder et al. (2016) pointed out that the identification and measurement of interactions within microbial community and accurate representation of these in theoretical models, presents an emerging challenge in microbial ecology, which the key objective is to reproduce temporal trajectories of the populations within the community.

This work presents the development of an aquatic microbial microcosm with an ecological structure, to evaluate the effects of contamination by toxic substances in the aquatic environment on the whole ecosystem at a small scale. The evaluation of the disturbances is based on the exposure of species, in terms of morphological groups, in a typical ecological succession, conducted by an organizational ecosystem approach. The quantification of the effects is given by the changes on the ecological community structure, according to the theoretical approach of Branco (1986) and Mckinney (2005).

\section{MATERIAL AND METHODS}

\section{Microbial ecology}

Microorganisms are important inhabitants of aquatic ecosystems, where they fulfill critical role in primary productivity, nutrient cycling, and decomposition (DeLorenzo et al., 2001). They have the potential for rapid growth and short generation times (Prosser et al., 2007), are easy to culture, provide rapid results and can be maintained under known controlled conditions (Mayfield, 2009). They are sensitive to environmental pollutants (Wang et al., 2011). Belonging to lower trophic levels, they can be warning indicators of potential damage to an aquatic ecosystem (Kelly \& Harwell, 1990). It is the simplicity of microbial model systems that makes them such powerful tools for the study of ecology (Jessup et al., 2004).

\section{Predictive model of whole ecosystems}

The goal is to develop a microbial model system to predict and evaluate, at the ecosystem level, the contamination of aquatic ecosystems by toxicants.

\section{Microcosm model description}

Favorable environment condition generates a microbial aquatic community, in a typical ecological succession, identified by simple microscopic methods.

\section{Temporal dynamics in microbial community}

After the identification of the microbial community structure and it's dynamics, it is possible study the ecological changes caused by the biological inhibitors introduced into the systems.

\section{Experimental design}

The substrate was placed in glass recipients with two liters and kept under continuous aeration done by compressors and tank vents. The air arrives into the systems by silicone hoses of $5 \mathrm{~mm}$ of diameter and scatters by a single diffuser. The containers were partially capped and maintained at room temperature.

a. Composition of the substrate: skimmed UHT milk nutritional composition $(100 \mathrm{~mL}): 3.18 \mathrm{~g}$ of proteins, $4.90 \mathrm{~g}$ of carbohydrates, $0.50 \mathrm{~g}$ of lipids, $0.10 \mathrm{~g}$ of dietary fiber, 141 $\mathrm{mg}$ of calcium, $94 \mathrm{mg}$ of phosphorus, $115 \mathrm{mg}$ of potassium and $50 \mathrm{mg}$ of sodium. b. Energy supply: $350 \mathrm{mg} \mathrm{L}^{-1}$ of BOD (diluted in distilled water). c. Period: 9 consecutive days. d. Sampling: 12 per each system every $24 \mathrm{~h}$ taken randomly with a glass pipette. e. Counting of microorganisms: by chamber of Sedwick-Rafter (optical microscope: 100$1000 \mathrm{x}$ ). f. Identification of microorganisms: according to Berk \& Gunderson (1993). g. Monitoring: pH, water temperature and dissolved oxygen (DO) h. Toxic agents: copper sulphate $\left(\mathrm{CuSO}_{4} .5 \mathrm{H}_{2} \mathrm{O}\right)$ and dichlorvos, DDVP-20 (dimethyl 2,2-dichlorovinyl phosphate), organophosphate insecticide. The choice was due their extensive use in many agricultural crops which produces waste that is swept by rain to the collections of water (Andrei, 2013). With particular importance in toxicological studies, the copper is also used in industrial activities and water quality control (Nikinmaa, 2014). i. Replications and disturbances: Experiments (E-1/E-2) with application of copper at concentrations: $\mathrm{C} 0=$ control, $\mathrm{C} 1=3.07 \mathrm{mg} \mathrm{L}^{-1} \mathrm{Cu}, \mathrm{C} 2=6.14 \mathrm{mg} \mathrm{L}^{-1} \mathrm{Cu}, \mathrm{C} 3=12.28 \mathrm{mg} \mathrm{L}^{-1}$ $\mathrm{Cu}$ (5 replicates as control +5 each with a concentration $=35$ microcosms). Experiment (E-3) with application of DDVP20: $\mathrm{C} 0=$ control, $\mathrm{C} 1=124 \mathrm{mg} \mathrm{L}^{-1}, \mathrm{C} 2=300 \mathrm{mg} \mathrm{L}^{-1}, \mathrm{C} 3=500 \mathrm{mg}$ $\mathrm{L}^{-1}(5$ replicates $=20$ microcosms $)$.

\section{RESULTS}

After $24 \mathrm{~h}$ of aeration, occurred the generation of filamentous bacteria, followed by free swimming ciliates protozoa, starting on the $3^{\text {rd }}$ day, reaching its maximum with logarithmic growth on the $4^{\text {th }}$ day. The treatment of toxic agents was made on the $4^{\text {th }}$ day to facilitate the quantification of the induced effects. Statistical analysis was performed by Kruskall-Wallis one-way ANOVA $\alpha=0.05$ (tables 1 to 6 ). 
The filamentous bacteria presented growth with predominance of short filaments. Each fragment was considered as a unit. The averages were in almost all microcosms around (2-15 units $\mathrm{mL}^{-1}$ ) excepting the microcosm control $\mathrm{M} 3 \mathrm{C} 0$ with more expressive numbers, 88 ( $5^{\text {th }}$ day) and 485 units $\mathrm{mL}^{-1},\left(9^{\text {th }}\right.$ day), (E-1/E-2). Some increase was suggested for copper at $3.07 \mathrm{mg} \mathrm{L}^{-1} \mathrm{Cu}$, microcosm M1C1, 46 (6 ${ }^{\text {th }}$ day) and 51 units/ $\mathrm{mL}$ ( $9^{\text {th }}$ day), (E-1) and at $300 \mathrm{mg} \mathrm{L}^{-1}$ of DDVP-20, microcosm M4C2, 203 ( $6^{\text {th }}$ day) and 74 units $/ \mathrm{mL}$, ( $9^{\text {th }}$ day).

The ciliates presented typical exponential growth, with averages around $700-1350$ ciliates $/ \mathrm{mL}$ with intermediate averages around $300-580$ ciliates $/ \mathrm{mL}(\mathrm{E}-1)$ on the $4^{\text {th }}$ day. Complete inhibitory effect of growth was suggested at 12.28 $\mathrm{mg} \mathrm{L}^{-1} \mathrm{Cu}$ and at $500 \mathrm{mg} \mathrm{L}^{-1}$ of DDVP 20. The results at $500 \mathrm{mg} \mathrm{L}^{-1}$ of DDVP-20 indicated some discrete recovery in 3 microcosms, MIC3, M2C3 and M4C3 with 175-90-83 ciliates $/ \mathrm{mL}$, respectively, on the $9^{\text {th }}$ day.

\section{DISCUSSION}

The quality of the substrate changed over time as succession progressed, being directly modified by the microorganisms, according to distinguishing features where this type of microbial succession is likely to be found as the organic wastewater bioreactors in the theoretical model described by Branco (1986) and McKinney (2005) and, as primary succession fueled by organic carbon derived from the substrate itself (Fierer et al., 2010).

The initial conditions favored the growth of filamentous bacteria able to consume food in dissolved form, followed by free swimming ciliates protozoa. To survive and grow, the bacteria must obtain soluble nutrients from the immediate environment (Branco, 1986).

The results of filamentous bacteria were discrepant, suggesting that there no was typical behavior and producing p-values inferior to $\alpha=0.05$ (tables 1,3 and 5).

Despite the averages were quite inexpressive, (2-15 units/ $\mathrm{mL}$ ), in almost all microcosms, excepting the microcosm M3C0 (88-485) units/ $\mathrm{mL}$ producing $\mathrm{p}=0.0290,5^{\text {th }}$ day $0.3027,9^{\text {th }}$ day $(\mathrm{E}-1)$ and $\mathrm{p}=0.0015,5^{\text {th }}$ day $-0.0056,9^{\text {th }}$ day (E-2), the results suggested variability (tables 1 and 3 ) and self-organization mechanism. Complete inhibitory effect of growth was suggested at $12.28 \mathrm{mg} / \mathrm{L}^{-1} \mathrm{Cu}, \mathrm{p}=0.0015,5^{\text {th }}$ day, (E-2) and DDVP-20 at $500 \mathrm{mg} / \mathrm{L}^{-1}, \mathrm{p}=0.0034,5^{\text {th }}$ day, (E-3), tables 3 and 5. Some increase was suggested for copper at $3.07 \mathrm{mg} \mathrm{L}^{-1} \mathrm{Cu}$ - microcosm M1C1, (46-51) units/ mL, ( $\mathrm{p}=$ $0.0078,6^{\text {th }}$ day $-\mathrm{p}=0.3027,9^{\text {th }}$ day $),(\mathrm{E}-1)$, table 1 , and DDVP20 at $300 \mathrm{mg} \mathrm{L}^{-1}$, microcosm M4C2, (203-74) units/ mL, $\left(\mathrm{p}=0.0124,6^{\text {th }}\right.$ day $\left.-\mathrm{p}=0.7633\right), 9^{\text {th }}$ day, table 5 .

About the predominance of short filaments, Pernthaler et al. (2004) pointed out that, at carrying capacity, (this method), the population converges to a predominance of short filaments. To (Rossetti et al., 2011), it is shown that changes in generation time can alter length distributions. We can only speculate which other parameter such as the substrate concentration, in this research of $350 \mathrm{mg} \mathrm{L}-1$ of BOD, (Teeling et al., 2012). In Fierer et al. (2010), the broad range of patterns of microbial primary succession is divided into three categories based on the source of carbon inputs.

Table 1. Growth dynamics of filamentous bacteria in substrate of $350 \mathrm{mg} \mathrm{L}^{-1}$ in continuous aeration at each three disturbances levels of copper sulphate on the $4^{\text {th }}$ day - (Experiment - 1).

\begin{tabular}{lcccccccccc}
\hline MICROCOSM & Day 0 & Day 1 & Day 2 & Day 3 & Day 4 & Day 5 & Day 6 & Day 7 & Day 8 & Day 9 \\
\hline (M1-C0) & 0.00 & 2.00 & 2.25 & 15.33 & 8.00 & 4.92 & 27.25 & 1.33 & 12.50 & 3.83 \\
(M2-C0) & 0.00 & 2.00 & 2.75 & 4.00 & 1.00 & 2.50 & 24.00 & 23.00 & 0.58 & 0.83 \\
(M3-C0) & 0.00 & 2.00 & 2.17 & 7.00 & 1.00 & 88.50 & 181.25 & 349.42 & 430.25 & 484.75 \\
(M4-C0) & 0.00 & 2.00 & 2.50 & 7.00 & 0.00 & 2.50 & 3.08 & 0.58 & 2.00 & 2.58 \\
(M5-C0) & 0.00 & 2.00 & 1.58 & 5.00 & 1.08 & 1.00 & 1.67 & 1.62 & 4.08 & 4.17 \\
(M1-C1) & 0.00 & 0.00 & 1.40 & 2.42 & 0.50 & 10.00 & 46.00 & 30.25 & 30.17 & 51.00 \\
(M2-C1) & 0.00 & 0.00 & 2.00 & 3.42 & 1.64 & 4.00 & 2.25 & 3.00 & 2.00 & 1.00 \\
(M3-C1) & 0.00 & 0.00 & 2.08 & 3.50 & 1.08 & 3.50 & 3.00 & 2.00 & 3.08 & 1.00 \\
(M4-C1) & 0.00 & 0.00 & 2.00 & 3.08 & 0.42 & 2.50 & 3.67 & 2.00 & 2.00 & 1.00 \\
(M5-C1) & 0.00 & 0.00 & 0.00 & 1.00 & 1.08 & 3.00 & 3.25 & 2.00 & 2.00 & 2.08 \\
(M1-C2) & 0.00 & 0.00 & 2.08 & 3.27 & 1.17 & 2.50 & 3.58 & 6.55 & 7.00 & 15.50 \\
(M2-C2) & 0.00 & 0.00 & 1.33 & 2.09 & 1.50 & 1.25 & 2.17 & 3.00 & 2.08 & 2.17 \\
(M3-C2) & 0.00 & 0.00 & 1.17 & 1.67 & 1.50 & 1.08 & 1.17 & 2.00 & 2.00 & 2.00 \\
(M4-C2) & 0.00 & 0.00 & 0.50 & 1.00 & 1.08 & 1.58 & 1.83 & 2.08 & 2.08 & 2.25 \\
(M5-C2) & 0.00 & 0.00 & 2.00 & 2.17 & 1.83 & 1.17 & 1.00 & 0.83 & 2.00 & 1.25 \\
(M1-C3) & 0.00 & 1.17 & 0.17 & 0.00 & 2.92 & 0.25 & 1.58 & 0.75 & 2.67 & 1.50 \\
(M2-C3) & 0.00 & 1.17 & 0.17 & 0.00 & 2.92 & 0.25 & 1.58 & 0.75 & 2.67 & 1.50 \\
(M3-C3) & 0.00 & 1.25 & 0.33 & 0.33 & 2.08 & 1.50 & 0.42 & 0.50 & 0.83 & 1.50 \\
(M4-C3) & 0.00 & 0.58 & 0.17 & 0.08 & 2.25 & 0.42 & 0.42 & 0.33 & 0.25 & 1.00 \\
(M5-C3) & 0.00 & 0.00 & 0.00 & 0.00 & 1.00 & 0.00 & 0.50 & 0.00 & 0.50 & 2.00 \\
\hline $\mathbf{p}$ & & $\mathbf{0 . 0 0 1 1}$ & $\mathbf{0 . 0 0 6 5}$ & $\mathbf{0 . 0 0 0 9}$ & $\mathbf{0 . 0 4 5 5}$ & $\mathbf{0 . 0 2 9 0}$ & $\mathbf{0 . 0 0 7 8}$ & $\mathbf{0 . 0 2 3 1}$ & $\mathbf{0 . 1 3 8 6}$ & $\mathbf{0 . 3 0 2 7}$ \\
\hline
\end{tabular}

Legend: $\mathrm{M}$ (microcosm); $\mathrm{C}=0$, control; $\mathrm{C} 1=3.07 \mathrm{mg} \mathrm{L}^{-1} \mathrm{Cu} ; \mathrm{C} 2=6.14 \mathrm{mgL}^{-1} \mathrm{Cu} ; \mathrm{C} 3=12.28 \mathrm{mg} \mathrm{L}^{-1} \mathrm{Cu}$ 
Table 2. Growth dynamics of free swimming ciliates protozoa in substrate of $350 \mathrm{mg} \mathrm{L}^{-1}$ in continuous aeration at each three disturbances levels of copper sulphate on the $4^{\text {th }}$ day - (Experiment - 1).

\begin{tabular}{lcccccccccc}
\hline MICROCOSM & Day 0 & Day 1 & Day 2 & Day 3 & Day 4 & Day 5 & Day 6 & Day 7 & Day 8 & Day 9 \\
\hline (M1-C0) & 0.00 & 0.00 & 0.00 & 8.08 & 960.25 & 494.33 & 295.50 & 155.25 & 74.00 & 39.17 \\
(M2-C0) & 0.00 & 0.00 & 0.00 & 35.92 & 1236.83 & 736.67 & 362.00 & 191.67 & 87.75 & 46.58 \\
(M3-C0) & 0.00 & 0.00 & 0.00 & 0.00 & 684.17 & 356.50 & 271.17 & 133.33 & 49.92 & 7.58 \\
(M4-C0) & 0.00 & 0.00 & 0.00 & 85.75 & 1153.08 & 717.83 & 428.33 & 266.92 & 106.75 & 59.00 \\
(M5-C0) & 0.00 & 0.00 & 0.00 & 16.17 & 1103.92 & 624.58 & 302.42 & 172.33 & 81.00 & 37.83 \\
(M1-C1) & 0.00 & 0.00 & 0.00 & 5.00 & 700.17 & 360.92 & 47.33 & 19.50 & 10.50 & 24.00 \\
(M2-C1) & 0.00 & 0.00 & 0.00 & 26.75 & 950.50 & 777.92 & 231.67 & 103.83 & 161.00 & 41.00 \\
(M3-C1) & 0.00 & 0.00 & 0.00 & 3.08 & 68.25 & 250.33 & 194.50 & 134.17 & 184.42 & 191.17 \\
(M4-C1) & 0.00 & 0.00 & 0.00 & 5.00 & 260.00 & 327.42 & 566.50 & 196.33 & 86.58 & 5.00 \\
(M5-C1) & 0.00 & 0.00 & 0.00 & 4.08 & 482.08 & 860.92 & 598.58 & 330.92 & 350.75 & 1.00 \\
(M1-C2) & 0.00 & 0.00 & 0.00 & 7.25 & 355.50 & 21.42 & 102.67 & 350.42 & 361.33 & 9.17 \\
(M2-C2) & 0.00 & 0.00 & 0.00 & 11.00 & 310.00 & 412.50 & 429.75 & 364.25 & 222.17 & 17.67 \\
(M3-C2) & 0.00 & 0.00 & 0.00 & 5.08 & 343.83 & 148.33 & 35.17 & 18.83 & 2.00 & 0.00 \\
(M4-C2) & 0.00 & 0.00 & 0.00 & 35.50 & 981.42 & 87.08 & 24.25 & 25.08 & 33.58 & 10.90 \\
(M5-C2) & 0.00 & 0.00 & 0.00 & 0.00 & 426.50 & 100.92 & 75.75 & 130.58 & 193.67 & 1.42 \\
(M1-C3) & 0.00 & 0.00 & 0.00 & 0.00 & 324.33 & 6.00 & 0.00 & 0.00 & 0.00 & 0.00 \\
(M2-C3) & 0.00 & 0.00 & 0.00 & 0.00 & 274.92 & 0.00 & 0.00 & 0.00 & 0.00 & 0.00 \\
(M3-C3) & 0.00 & 0.00 & 0.00 & 11.92 & 502.00 & 10.00 & 0.00 & 12.00 & 0.00 & 0.00 \\
(M4-C3) & 0.00 & 0.00 & 0.00 & 173.92 & 653.92 & 0.00 & 0.00 & 0.00 & 0.00 & 0.00 \\
(M5-C3) & 0.00 & 0.00 & 0.00 & 301.75 & 814.92 & 0.00 & 0.00 & 0.00 & 0.00 & 0.00 \\
\hline p & & & & $\mathbf{0 . 6 8 3 6}$ & $\mathbf{0 . 0 4 4 5}$ & $\mathbf{0 . 0 0 2 2}$ & $\mathbf{0 . 0 0 5 3}$ & $\mathbf{0 . 0 1 2 1}$ & $\mathbf{0 . 0 1 0 4}$ & $\mathbf{0 . 0 0 7 2}$ \\
\hline
\end{tabular}

Legend: $\mathrm{M}$ (microcosm); $\mathrm{C}=0$, control; $\mathrm{C} 1=3.07 \mathrm{mg} \mathrm{L}^{-1} \mathrm{Cu} ; \mathrm{C} 2=6.14 \mathrm{mgL}^{-1} \mathrm{Cu} ; \mathrm{C} 3=12.28 \mathrm{mg} \mathrm{L}^{-1} \mathrm{Cu}$

Table 3. Growth dynamics of filamentous bacteria in substrate of $350 \mathrm{mg} \mathrm{L}^{-1}$ in continuous aeration at each three disturbances levels of copper sulphate on the $4^{\text {th }}$ day-(Experiment -2$)$.

\begin{tabular}{lcccccccccc}
\hline MICROCOSM & Day 0 & Day 1 & Day 2 & Day 3 & Day 4 & Day 5 & Day 6 & Day 7 & Day 8 & Day 9 \\
\hline (M1-C0) & 0.00 & 2.00 & 2.25 & 15.33 & 8.00 & 4.92 & 27.25 & 1.33 & 12.50 & 3.83 \\
(M2-C0) & 0.00 & 2.00 & 2.75 & 4.00 & 1.00 & 2.50 & 2.58 & 0.00 & 0.58 & 0.83 \\
(M3-C0) & 0.00 & 2.00 & 2.17 & 7.00 & 1.00 & 88.50 & 181.25 & 349.42 & 430.25 & 484.75 \\
(M4-C0) & 0.00 & 2.00 & 2.50 & 7.00 & 0.00 & 2.50 & 3.08 & 0.58 & 2.00 & 2.58 \\
(M5-C0) & 0.00 & 2.00 & 1.58 & 5.00 & 1.08 & 1.00 & 1.67 & 1.67 & 4.08 & 4.17 \\
(M1-C1) & 0.00 & 0.00 & 4.08 & 2.42 & 4.08 & 10.00 & 2.00 & 1.33 & 1.42 & 1.08 \\
(M2-C1) & 0.00 & 3.33 & 3.25 & 3.42 & 1.08 & 7.42 & 3.50 & 2.67 & 7.67 & 0.75 \\
(M3-C1) & 0.00 & 4.08 & 2.08 & 4.00 & 1.00 & 11.50 & 9.08 & 2.00 & 5.17 & 1.00 \\
(M4-C1) & 0.00 & 1.00 & 2.00 & 3.08 & 1.08 & 5.58 & 2.58 & 2.00 & 4.75 & 1.00 \\
(M5-C1) & 0.00 & 2.58 & 1.83 & 5.00 & 0.17 & 4.75 & 1.50 & 2.33 & 2.00 & 2.08 \\
(M1-C2) & 0.00 & 2.00 & 2.08 & 3.27 & 2.00 & 0.00 & 3.00 & 0.50 & 1.00 & 0.33 \\
(M2-C2) & 0.00 & 1.83 & 1.33 & 2.09 & 0.00 & 0.00 & 0.00 & 1.17 & 2.08 & 1.67 \\
(M3-C2) & 0.00 & 2.08 & 1.17 & 4.08 & 0.00 & 0.00 & 0.00 & 1.00 & 0.00 & 1.00 \\
(M4-C2) & 0.00 & 2.42 & 6.00 & 0.00 & 0.00 & 0.00 & 0.17 & 0.00 & 0.00 & 0.00 \\
(M5-C2) & 0.00 & 2.08 & 1.00 & 2.08 & 0.00 & 0.17 & 0.25 & 0.00 & 0.00 & 1.25 \\
(M1-C3) & 0.00 & 1.42 & 3.33 & 5.00 & 0.00 & 0.00 & 0.00 & 0.25 & 0.50 & 0.00 \\
(M2-C3) & 0.00 & 1.25 & 1.50 & 0.00 & 0.08 & 0.00 & 0.00 & 0.00 & 0.00 & 0.00 \\
(M3-C3) & 0.00 & 1.25 & 1.42 & 7.08 & 0.00 & 0.00 & 0.00 & 0.50 & 0.00 & 0.00 \\
(M4-C3) & 0.00 & 0.92 & 4.25 & 3.08 & 0.00 & 0.17 & 0.00 & 0.00 & 0.00 & 0.00 \\
(M5-C3) & 0.00 & 0.00 & 0.00 & 0.00 & 0.00 & 0.00 & 0.00 & 0.00 & 0.00 & 0.00 \\
\hline p & & $\mathbf{0 . 0 2 7 4}$ & $\mathbf{0 . 3 5 5 7}$ & $\mathbf{0 . 1 3 4 3}$ & $\mathbf{0 . 0 3 4 4}$ & $\mathbf{0 . 0 0 1 5}$ & $\mathbf{0 . 0 0 6}$ & $\mathbf{0 . 0 1 2 8}$ & $\mathbf{0 . 0 0 7 4}$ & $\mathbf{0 . 0 0 5 6}$ \\
\hline
\end{tabular}

Legend: $\mathrm{M}$ (microcosm); $\mathrm{C}=0$, control; $\mathrm{C} 1=3.07 \mathrm{mg} \mathrm{L}^{-1} \mathrm{Cu} ; \mathrm{C} 2=6.14 \mathrm{mgL}^{-1} \mathrm{Cu} ; \mathrm{C} 3=12.28 \mathrm{mg} \mathrm{L}^{-1} \mathrm{Cu}$

In contrast, the ciliates presented significant exponential growth suggesting predictable and typical behavior, which starting on the $3^{\text {th }}$ day, produced $\mathrm{p}$-values superior to $\alpha=0.05: 0.6836(\mathrm{E}-1), 0.8583(\mathrm{E}-2)$ and $0.5977(\mathrm{E}-3)$ reaching its maximum, with averages of $700-1350$ ciliates/
$\mathrm{mL}$ with intermediate levels of $300-580$ ciliates/ $\mathrm{mL}$, producing $\mathrm{p}=0.0445(\mathrm{E}-1), \mathrm{p}=0.05365(\mathrm{E}-2)$ and $\mathrm{p}=0.5142$ (E-3) on the $4^{\text {th }}$ day, although some variability and selforganization mechanism was suggested in the microcosms, tables 2, 4 and 6 . 
Table 4. Growth dynamics of free swimming ciliates protozoa in substrate of $350 \mathrm{mg} \mathrm{L}^{-1}$ in continuous aeration at each three disturbances levels of copper sulphate on the $4^{\text {th }}$ day - (Experiment 4$)$.

\begin{tabular}{lcccccccccc}
\hline MICROCOSM & Day 0 & Day 1 & Day 2 & Day 3 & Day 4 & Day 5 & Day 6 & Day 7 & Day 8 & Day 9 \\
\hline (M1-C0) & 0.00 & 0.00 & 0.00 & 8.08 & 960.25 & 494.33 & 295.50 & 155.25 & 74.00 & 39.17 \\
(M2-C0) & 0.00 & 0.00 & 0.00 & 35.92 & 1236.83 & 736.67 & 362.00 & 191.67 & 87.75 & 46.58 \\
(M3-C0) & 0.00 & 0.00 & 0.00 & 0.00 & 684.17 & 356.50 & 271.17 & 133.33 & 49.92 & 7.58 \\
(M4-C0) & 0.00 & 0.00 & 0.00 & 85.75 & 1153.08 & 736.67 & 428.33 & 266.92 & 106.75 & 59.00 \\
(M5-C0) & 0.00 & 0.00 & 0.00 & 16.17 & 1103.92 & 624.58 & 302.42 & 172.33 & 81.00 & 37.83 \\
(M1-C1) & 0.00 & 0.00 & 0.00 & 5.00 & 718.92 & 328.83 & 130.92 & 19.50 & 9.42 & 24.00 \\
(M2-C1) & 0.00 & 0.00 & 0.00 & 26.75 & 897.25 & 401.42 & 185.67 & 100.58 & 66.08 & 41.00 \\
(M3-C1) & 0.00 & 0.00 & 0.00 & 3.08 & 1079.25 & 383.83 & 260.42 & 163.50 & 190.92 & 191.92 \\
(M4-C1) & 0.00 & 0.00 & 0.00 & 5.00 & 974.83 & 373.08 & 150.92 & 196.33 & 26.92 & 5.00 \\
(M5-C1) & 0.00 & 0.00 & 0.00 & 4.08 & 916.83 & 354.58 & 127.17 & 310.25 & 78.00 & 6.83 \\
(M1-C2) & 0.00 & 0.00 & 0.00 & 0.00 & 788.33 & 193.83 & 53.25 & 228.00 & 64.67 & 9.17 \\
(M2-C2) & 0.00 & 0.00 & 0.00 & 0.00 & 1279.58 & 245.92 & 86.50 & 164.50 & 24.58 & 17.67 \\
(M3-C2) & 0.00 & 0.00 & 0.00 & 18.17 & 1010.08 & 197.50 & 40.17 & 18.83 & 2.00 & 0.00 \\
(M4-C2) & 0.00 & 0.00 & 0.00 & 35.50 & 962.58 & 152.17 & 137.33 & 47.00 & 13.17 & 10.9 \\
(M5-C2) & 0.00 & 0.00 & 0.00 & 15.67 & 1162.58 & 228.67 & 74.83 & 94.08 & 134.25 & 0.00 \\
(M1-C3) & 0.00 & 0.00 & 0.00 & 0.00 & 784.08 & 59.08 & 13.25 & 0.00 & 0.00 & 0.00 \\
(M2-C3) & 0.00 & 0.00 & 0.00 & 0.00 & 1140.42 & 67.83 & 6.81 & 0.00 & 0.00 & 0.00 \\
(M3-C3) & 0.00 & 0.00 & 0.00 & 0.00 & 1240.92 & 40.92 & 8.17 & 12.00 & 0.00 & 0.00 \\
(M4-C3) & 0.00 & 0.00 & 0.00 & 158.33 & 945.17 & 8.00 & 7.42 & 0.00 & 0.00 & 0.00 \\
(M5-C3) & 0.00 & 0.00 & 0.00 & 258.92 & 919.67 & 18.58 & 2.83 & 0.00 & 0.00 & 0.00 \\
\hline $\mathbf{p}$ & & & & $\mathbf{0 . 8 5 8 3}$ & $\mathbf{0 . 0 5 3 6 5}$ & $\mathbf{0 . 0 0 7}$ & $\mathbf{0 . 0 0 6}$ & $\mathbf{0 . 0 0 8 6}$ & $\mathbf{0 . 0 0 7 1}$ & $\mathbf{0 . 0 0 9 1}$ \\
\hline
\end{tabular}

Legend: $\mathrm{M}$ (microcosm); $\mathrm{C}=0$, control; $\mathrm{C} 1=3.07 \mathrm{mg} \mathrm{L}^{-1} \mathrm{Cu} ; \mathrm{C} 2=6.14 \mathrm{mgL}^{-1} \mathrm{Cu} ; \mathrm{C} 3=12.28 \mathrm{mg} \mathrm{L}^{-1} \mathrm{Cu}$

Table 5. Growth dynamics of filamentous bacteria in substrate of $350 \mathrm{mg} \mathrm{L}^{-1}$ of BOD in continuous aeration at each three disturbances levels of dichlorvos 20 on the $4^{\text {th }}$ day - (Experiment -5$)$.

\begin{tabular}{lcccccccccc}
\hline MICROCOSM & Day 0 & Day 1 & Day 2 & Day 3 & Day 4 & Day 5 & Day 6 & Day 7 & Day 8 & Day 9 \\
\hline (M1-C0) & 0.00 & 1.50 & 2.92 & 9.08 & 7.67 & 0.75 & 2.00 & 1.17 & 1.58 & 1.00 \\
(M2-C0) & 0.00 & 2.00 & 5.08 & 3.00 & 2.08 & 4.08 & 1.25 & 0.92 & 1.08 & 1.92 \\
(M3-C0) & 0.00 & 2.08 & 2.67 & 5.17 & 4.08 & 6.08 & 3.08 & 1.00 & 1.25 & 0.00 \\
(M4-C0) & 0.00 & 2.00 & 3.83 & 10.08 & 4.00 & 6.08 & 0.67 & 1.83 & 2.00 & 1.42 \\
(M5-C0) & 0.00 & 2.67 & 3.08 & 5.50 & 4.08 & 6.08 & 30.33 & 13.33 & 7.50 & 7.00 \\
(M1-C1) & 0.00 & 0.00 & 4.92 & 5.17 & 4.33 & 22.17 & 6.08 & 4.00 & 4.00 & 4.25 \\
(M2-C1) & 0.00 & 3.33 & 3.25 & 6.25 & 6.00 & 10.25 & 6.00 & 5.83 & 6.00 & 3.50 \\
(M3-C1) & 0.00 & 2.58 & 2.08 & 3.75 & 0.50 & 6.17 & 3.08 & 3.50 & 4.42 & 6.25 \\
(M4-C1) & 0.00 & 1.00 & 2.00 & 3.08 & 1.08 & 5.58 & 10.58 & 3.50 & 0.92 & 0.67 \\
(M5-C1) & 0.00 & 2.25 & 2.00 & 5.33 & 2.00 & 5.92 & 5.33 & 2.33 & 2.00 & 2.08 \\
(M1-C2) & 0.00 & 2.00 & 2.08 & 3.27 & 15.17 & 14.25 & 5.50 & 2.33 & 0.92 & 1.58 \\
(M2-C2) & 0.00 & 1.83 & 1.33 & 2.09 & 3.08 & 8.00 & 23.75 & 10.17 & 2.25 & 1.75 \\
(M3-C2) & 0.00 & 2.08 & 1.17 & 4.08 & 2.00 & 6.33 & 0.92 & 0.50 & 0.83 & 0.83 \\
(M4-C2) & 0.00 & 1.33 & 2.42 & 6.00 & 0.00 & 10.67 & 203.42 & 77.75 & 66.92 & 74.42 \\
(M5-C2) & 0.00 & 2.08 & 1.00 & 2.08 & 0.00 & 17.08 & 0.25 & 0.00 & 1.25 & 0.83 \\
(M1-C3) & 0.00 & 1.83 & 2.00 & 4.50 & 2.17 & 0.00 & 0.00 & 0.25 & 0.50 & 7.00 \\
(M2-C3) & 0.00 & 1.58 & 3.17 & 3.67 & 1.33 & 0.08 & 0.00 & 0.00 & 1.17 & 5.25 \\
(M3-C3) & 0.00 & 1.92 & 2.33 & 4.17 & 4.33 & 0.00 & 0.00 & 0.50 & 0.00 & 0.75 \\
(M4-C3) & 0.00 & 1.00 & 1.83 & 2.00 & 1.92 & 0.00 & 0.00 & 0.00 & 0.00 & 0.00 \\
(M5-C3) & 0.00 & 1.25 & 0.92 & 2.33 & 0.92 & 0.08 & 0.00 & 0.42 & 0.00 & 0.00 \\
\hline $\mathbf{p}$ & & $\mathbf{0 . 3 3 2 1}$ & $\mathbf{0 . 0 4 4 2}$ & $\mathbf{0 . 1 3 3 9}$ & $\mathbf{0 . 4 2 4}$ & $\mathbf{0 . 0 0 3 4}$ & $\mathbf{0 . 0 1 2 4}$ & $\mathbf{0 . 0 2 2 6}$ & $\mathbf{0 . 2 6 8}$ & $\mathbf{0 . 7 6 3 3}$ \\
\hline
\end{tabular}

Legend: $\mathrm{M}$ (microcosm); $\mathrm{C} 0=$ control; $\mathrm{C} 1=124 \mathrm{mg} \mathrm{L}^{-1}$ of DDVP-20; $\mathrm{C} 2=300 \mathrm{mg} \mathrm{L}^{-1}$ of DDVP20; $\mathrm{C} 3=500 \mathrm{mg} \mathrm{L}^{-1}$ of DDVP-20

Despite the p-values (inferior $\alpha=0.05$ ), qualitatively, complete inhibitory effect of growth of ciliates was suggested at $12.28 \mathrm{mg} \mathrm{L}^{-1} \mathrm{Cu}$, producing $\mathrm{p}=0.0022(\mathrm{E}-1), \mathrm{p}=0.007(\mathrm{E}-$ 2) and at $500 \mathrm{mg} \mathrm{L}^{-1}$ of DDVP $20, \mathrm{p}=0.0007$ (E-3) ( $5^{\text {th }}$ day), with some recovery in 3 microcosms: $\mathrm{MIC} 3, \mathrm{M} 2 \mathrm{C} 3$ and
$\mathrm{M} 4 \mathrm{C} 3,(\mathrm{p}=0.1224),\left(9^{\text {th }}\right.$ day $)$, with $175-80-83$ ciliates $/ \mathrm{mL}$, respectively.

In Oviedo et al. (2002) at $2 \mathrm{mg} \mathrm{L}^{-1} \mathrm{Cu}$ on a continuous flow wastewater treatment, the growth of filamentous bacteria was significantly inhibited. Regarding to ciliates, the results can be 
Table 6. Growth dynamics of free swimming ciliates protozoa in substrate of $350 \mathrm{mg} \mathrm{L}^{-1}$ of BOD in continuous aeration at each three disturbances levels of dichlorvos 20 on the $4^{\text {th }}$ day - (Experiment - 6).

\begin{tabular}{|c|c|c|c|c|c|c|c|c|c|c|}
\hline MICROCOSM & Day 0 & Day 1 & Day 2 & Day 3 & Day 4 & Day 5 & Day 6 & Day 7 & Day 8 & Day 9 \\
\hline$(\mathrm{M} 1-\mathrm{C} 0)$ & 0.00 & 0.00 & 0.00 & 655.75 & 1259.42 & 496.25 & 291.92 & 181.50 & 90.67 & 51.42 \\
\hline (M2-C0) & 0.00 & 0.00 & 0.00 & 628.08 & 857.92 & 366.25 & 248.58 & 218.67 & 153.08 & 113.33 \\
\hline (M3-C0) & 0.00 & 0.00 & 0.00 & 259.67 & 1133.00 & 449.50 & 316.58 & 169.67 & 67.83 & 41.08 \\
\hline (M4-C0) & 0.00 & 0.00 & 0.00 & 833.58 & 1202.33 & 741.50 & 342.17 & 221.42 & 51.92 & 31.50 \\
\hline (M5-C0) & 0.00 & 0.00 & 0.00 & 177.00 & 935.00 & 658.75 & 454.58 & 252.08 & 95.33 & 51.83 \\
\hline (M1-C1) & 0.00 & 0.00 & 0.00 & 317.42 & 1350.67 & 149.00 & 199.25 & 60.25 & 23.67 & 22.17 \\
\hline (M2-C1) & 0.00 & 0.00 & 0.00 & 662.08 & 713.92 & 361.92 & 247.08 & 139.00 & 71.25 & 32.92 \\
\hline (M3-C1) & 0.00 & 0.00 & 0.00 & 588.18 & 809.67 & 246.83 & 130.42 & 60.58 & 39.25 & 35.50 \\
\hline (M4-C1) & 0.00 & 0.00 & 0.00 & 668.83 & 744.00 & 246.83 & 159.50 & 123.33 & 63.25 & 42.25 \\
\hline (M5-C1) & 0.00 & 0.00 & 0.00 & 329.58 & 1164.42 & 310.75 & 144.25 & 164.25 & 160.42 & 192.58 \\
\hline (M1-C2) & 0.00 & 0.00 & 0.00 & 227.42 & 900.58 & 143.42 & 102.17 & 124.75 & 131.17 & 145.17 \\
\hline (M2-C2) & 0.00 & 0.00 & 0.00 & 656.25 & 1180.50 & 89.92 & 159.67 & 139.75 & 146.58 & 149.00 \\
\hline (M3-C2) & 0.00 & 0.00 & 0.00 & 534.83 & 1091.17 & 219.58 & 165.17 & 163.92 & 164.00 & 168.58 \\
\hline (M4-C2) & 0.00 & 0.00 & 0.00 & 546.75 & 962.50 & 89.00 & 22.83 & 65.67 & 133.25 & 149.92 \\
\hline (M5-C2) & 0.00 & 0.00 & 0.00 & 826.08 & 1151.17 & 260.50 & 51.42 & 90.20 & 115.58 & 123.92 \\
\hline (M1-C3) & 0.00 & 0.00 & 0.00 & 510.33 & 1019.83 & 0.00 & 0.00 & 25.33 & 76.75 & 175.25 \\
\hline (M2-C3) & 0.00 & 0.00 & 0.00 & 903.67 & 582.50 & 0.00 & 0.00 & 6.75 & 47.67 & 89.33 \\
\hline (M3-C3) & 0.00 & 0.00 & 0.00 & 505.58 & 702.83 & 0.00 & 0.00 & 0.00 & 0.00 & 0.00 \\
\hline (M4-C3) & 0.00 & 0.00 & 0.00 & 852.83 & 940.75 & 0.00 & 0.00 & 0.00 & 30.00 & 83.08 \\
\hline (M5-C3) & 0.00 & 0.00 & 0.00 & 855.33 & 1176.00 & 0.00 & 0.00 & 0.00 & 0.00 & 0.00 \\
\hline $\mathbf{p}$ & & & & 0.5977 & 0.5142 & 0.0007 & 0.0009 & 0.001 & 0.0171 & 0.1224 \\
\hline
\end{tabular}

Legend: $\mathrm{M}$ (microcosm); $\mathrm{C} 0=$ control; $\mathrm{C} 1=124 \mathrm{mg} \mathrm{L}^{-1}$ of DDVP-20; $\mathrm{C} 2=300 \mathrm{mg} \mathrm{L}^{-1}$ of DDVP20; $3=500 \mathrm{mg} \mathrm{L}^{-1}$ of DDVP-20

comparable to the study of protozoa and metazoan communities when $8 \mathrm{mg} \mathrm{L}^{-1} \mathrm{Cu}$ caused little effects, with recovery within a week, and $20 \mathrm{mg} \mathrm{L}^{-1} \mathrm{Cu}$ caused drastic effect (Nicolau et al., 2007). To Stauber \& Davies (2000), there is an urgent need for more relevant tests to detect bioavailable copper guidelines in the environment including protozoa and bacteria.

Organophosphates insecticides, as a class, have adverse direct effects on bacteria and on protozoa. The direct effects on bacteria have been less consistent and at concentrations that would be expected within the environment, they have not produced any direct effects. When environmentally realistic concentrations were exceeded, negative and positive effects were observed (Staley et al., 2015).

The toxicity of insecticides, to bacteria and protozoa, is an area of research requiring further study and data are limited existing a great deal of variability (DeLorenzo et al., 2001). In DeLorenzo et al. (1999), $0.01 \mathrm{mg} / \mathrm{L}$ chlorpyrifos reduced ciliate abundance of a natural microbial community and an increase in bacterial abundance. In Staley et al. (2015), the study revealed mixed or no direct effects on bacterial species among all pesticides categories, chlorpyrifos increased abundance of bacterial species at $3300-9900 \mathrm{mg} \mathrm{L}^{-1}$ and malathion produced direct negative effects on ciliate protozoa at $1-30 \mathrm{mg} \mathrm{L}^{-1}$.

In Pratt et al. (1993), experiments using microcosms, developed from natural microbial communities, showed that microcosm variability was sufficiently low to detect adverse effects on species richness. The study revealed no significant effects of organophosphate pesticide even when concentrations exceeded water quality criteria threefold $(\sim 12$ $\mathrm{mg} \mathrm{L} \mathrm{L}^{-1}$ ). Copper reduced species richness and biomass at 0.01 to $0.020 \mathrm{mg} \mathrm{L}^{-1} \mathrm{Cu}$.

To (Ghiglione et al., 2106), microorganisms present the capability to cope with chemical and/or biological contaminants to restore polluted sites. Staley et al. (2015) pointed out also that few studies have been conducted to specifically address community-level effects of pesticides on microorganisms, and more research is necessary to better understand and predict the net effects of pesticides on ecosystem health.

The systems presented temperatures of $\left(20^{\circ} \mathrm{C}-24^{\circ} \mathrm{C}\right)$, above $35^{\circ} \mathrm{C}$ could cause denaturation; $\mathrm{pH}$ from 7.0 ( $1^{\text {st }}$ day) to 8.0 ( $9^{\text {th }}$ day), values below 4.0 and above 9.5 may trigger toxic effect and dissolved oxygen (DO) of $\left(5.2 \mathrm{mg} \mathrm{L}^{-1}-6.2 \mathrm{mg} \mathrm{L}^{-1}\right)$, were satisfactory (McKinney, 2005).

The microcosms suggested self-organization mechanism of ecological system and typical stages of succession. The concept of self-organization is defined as the evolution of a system into an organized form in the absence of external pressures. The self-organization process by which ecosystem develops structure, functions, and diversity from available energies and matter is called succession (Odum \& Barrett, 2014).

The variability that occurs in similar experiments has been the object of development of models (Beyers \& Odum, 1993). Differences between microcosm responses and toxicity based on standard bioassays are a result of complex interactions including toxicant degradation, the interaction of toxicants and nutrients, and the lack of species sensitive to some toxicants (Pratt et al, 1993). Yet, as the variability could reduce the demonstration of the effects, qualitative analysis is 
more indicated (Boudou \& Ribeyre, 1989).

To Widder et al. (2016), because of the rapid growth rates of microorganisms, selection pressures can be lead to different responses in bioassay. Curtis et al. (2009) consider the evolution as the master variable. Brandt et al., (2004) also admit that stochastic events involved in the succession of natural microbial communities may introduce difficulties in performing controlled replicated experiments, but the advantages of using naturally complex and metabolically diverse populations overshadow this latent error.

\section{Caveats and ambitions}

Understanding the ecology of microorganisms is arguably one of the most compelling intellectual challenges facing contemporary ecology (Prosser et al., 2007).The challenge is further develop theoretical and methodological features while at the same time contributing to current pressing problems such as the management of global water resources (Psenner et al., 2008). Ghiglione et al. (2016) stressed that the microbial ecotoxicology paves the way to assess the impacts of contaminants on taxonomic and functional microbial biodiversity which is supporting ecosystem functions and ensuring their stability and recovery.

The practical application motivated the methodology studied. The BOD was close to that of the organic waste commonly discharged into the water. As closed system, the results can be compared only to the point samples of aquatic ecosystems. The method should be indicated only for the evaluation of contaminants in the microbial community at a given moment, which would correspond to the effect of a given effluent, immediately after its discharge into the water. Given the various sensitivities of different microorganisms to toxic chemicals there is a growing interest in microbial toxicity testing at the community or ecosystem level (Brandt et al., 2004). The key to achieving this level of predictive understanding is the integrative development of mathematical models, method and data collection (Widder et al., 2016).

\section{CONCLUSION}

The experimental model provided an aquatic microbial community structure, composed by filamentous bacteria, followed by free swimming protozoa ciliates in a typical ecological succession. Ciliates, presented exponential growth on the $4^{\text {th }}$ day with significant predictable behavior and complete inhibitory effect of growth at concentrations of $12.28 \mathrm{mg} \mathrm{L}^{-1} \mathrm{Cu}$ and $500 \mathrm{mg} \mathrm{L}^{-1}$ DDVP-20. The variability verified in the microcosms suggested self-organization mechanism of ecological systems and qualitative analysis. Results of filamentous bacteria were inconclusive. Greater precision can be obtained with molecular biodiversity approach, development of opened system interrogating function-structure relation as well the integration between theory and data with the construction of mathematical models with pertinent parameters.

\section{ACKNOWLEGDEMENT}

Financial support was provided by São Paulo Research Foundation - FAPESP. I am grateful to Prof. Samuel Murgel Branco for his advice and inspiration. I also express my gratitude to Prof. Aristides Almeida Rocha and Prof. Tom P. Curtis for technical cooperation and enthusiasm. In honor of my parents, Yolanda and José.

\section{REFERENCES}

ANDREI, E. 2013. Compendio de defensivos agrícolas. Organização Andrei (ed), 1380p.

BERK, S. G. \& GUNDERSON, J. H. 1993. Wastewater organisms: a color atlas. CRC Press, 48p.

BEYERS, R. J. \& ODUM, H.T. 1993. Ecological Microcosms. Springer-Verlag, $572 p$.

BOUDOU, A. \& RIBEYRE, F.1989. Aquatic Ecotoxicology: fundamentals, concepts and methodologies. CRC Press, $352 \mathrm{p}$.

BRANCO, S. M. 1986. Hidrobiologia aplicada à engenharia sanitária. CETESB, 620p.

BRANDT, K.K., JøRGENSEN, N.O.G., NIESLEN, T.H. \& WINDING, A. 2004. Microbial community-level toxicity testing of linear alkylbenzene sulfonates in aquatic microcosms. FEMS Microbiol. Ecol. 49 (2) 229-241. http://dx.doi.org/10.1016/j. femsec.2004.03.006

CAIRNS, J. Jr. \& NIEDERLEHNER, B. R. 1994. Ecology toxicity testing: scale, complexity, and relevance. Cairns J.Jr. \& Niederlehner, B.R. (eds). Lewis Publishers, 240p.

CURTIS, T.P. 2007. Theory and the microbial world. Environ. Microbiol. 9(1)1-11. http://dx.doi.org/10.1111/j.14622920.2006.01222_1.x

CURTIS, T.P., WALLLBRIDGE, N.C. \& SLOAN, W.T. 2009. Theory, community assembly, diversity and evolution in the microbial world in Speciation and Patterns of Diversity. Butilin, R. K., Bridle, J.R. \& Schulter, D. (eds). Cambridge Univ. Press, pp.59-76.

COLWELL, R.R. 2012. Toxic effect of pollutants on microorganisms in Principles of Ecotoxicology. Walker, C.H., Sibly, R.M., Hopkin, S.P., Peakall, D.B. (eds). CRC Press, pp. 275-94.

DeLORENZO, M.E., SCOTT, G.I. \& ROSS, P.E. 1999. Effects of the agricultural pesticides atrazine, deethylatrazine, endosulfan and chlorpyrifos on an estuarine microbial food web. Environ. Toxicol. Chem.18(12):2824-35. http://dx.doi.org/ 10.1002/ etc. 5620181224

DeLORENZO, M.E., SCOTT, G.I. \& ROSS, P.E. 2001. Toxicity of pesticides to aquatic microorganisms: A review. Environ. Toxicol. Chem.Chem. 20(1):84-98. http://dx.doi.org/ 10.1002/ etc. 5620200108

FIERER, N., NEMERGUT, D., KINGHT R. \& CRAINE, J.M. 2010. Changes through time: integrating microorganisms into the study of succession. Res. Microbiol. 161(8):635-42. http://dx.doi. org/10.1016/j.resmic.2010.06.002

FISKESJÖ, G. 1993. The Allium test in wastewater monitoring. Environ. Toxicol. Water Qual. 8(3)291-8. http://dx.doi. org/10.1002/tox.2530080306

GHIGLIONE, J.F., LAURENT, F.M \& PESCE, S. 2016. Microbial ecotoxicology: an emerging discipline facing contemporary environmental threats. Environ Sci. Pollut. Res. 23(5):3981-83. http://dx.doi.org/10.1007/s11356-015-5763-1

JESSUP, C.M., KASSEN, R., FORDE, S.E., KERR, B. \& BUCKLING, A. 2004. Big questions, small worlds: microbial model systems in ecology. Trends Ecol. Evol. 19(4):189-97. 
http://dx.doi.org/10.1016/j.tree.2004.01.008

KELLY, J.R \& HARWELL, M.A. 1990. Indicators of ecosystem recovery. Environ. Manage. 14(5):527-545.htt://dx.doi. org/10.1007/BF02394708

LOGAR, R.M. \& VODOVNIK, M. 2007. The applications of microbes in environmental monitoring. Environ. Microbiol. In: Méndez-Vilas (eds) United States. Communicating current research and educational topics in applied microbiology, pp. 577-85.

MAYFIELD, C. I. 1994. Microbial systems, UK. In: Calow, P.P. (ed), UK, Handbook of toxicology. UK: Blackwell Science, pp.9-27.

McKINNEY, R.E., 2005. Environmental Pollution Control Microbiology: A fifty-year perspectives. Marcel Dekker Inc., $448 \mathrm{p}$.

NICOLAU, A. MOTA, M. \& LIMA, N. 2007. Microfauna as indicator of copper, zinc and

cycloheximide in activated sludge processes. Environ. Eng. Sci. 24(4):434-45. http://dx.doi.org/10.1089/ees.2006.06-0005

NIKINMAA, M. 2014. An Introduction to Aquatic Toxicology. Elsevier, 240p.

ODUM, E. P. \& BARRETT, G. W. 2014. Fundamentos de Ecologia. CENGAGE, 612p.

OVIEDO, M.D.C., MÁRQUEZ, J. S. \& ALONSO, M. 2002. Toxic effects of metals on microbial activity in the activated sludge process. Chem. Biochem. Eng. Q. 16 (3) 139-144.

PERNTHALER, J., ZÖLLNER, E., WARNECKE, F. \& JURGENS, K. 2004. Bloom of filamentous bacteria in a mesotrophic lake: identity and potential controlling mechanism. Appl. Environ. Microbiol. 70(10): 6272-81. http://dx.doi.org/10.1128/ AEM.70.10.6272-6281.2004

PRATT, J.R., BOWERS, N.J. \& BALCZON, J.M. 1993. A microcosm using naturally derived microbial communities: comparative ecotoxicology. In: Wayne, G. L., Hughes, J.S. and Lewis, M.A. (eds), Pennsylvania. Environmental Toxicology and Risk Assessment, Philadelphia, ASTM, 179, pp. 178-91. http://dx.doi.org/10.1520/STP19241S

PSENNER, R., ALFREIDER, A. \& SCHWARZ, A. 2008. Aquatic microbial ecology: water desert, microcosm, ecosystem. What's the next? Int. Rev. Hydrobiol. 93(4-5): 606-623. http://dx.doi. org/10.1002/iroh.200711044
PROSSER, J.I., BOHANNAN, B. J. M., CURTIS, T. P. \& ELLIS, R. J. 2007. The role of ecological theory in microbial ecology. Nat. Rev. Microbiol. (5):384-92. http://dx.doi.org /10.1038/ nrmicro1643

RAND, G.M. 1995. Fundamentals of aquatic toxicology: Effects, environmental fate and risk assessment, $2^{\text {nd }}$ ed. CRC Press, $1148 \mathrm{p}$.

RIBEIRO, I.A. 1998. Teste com raízes de cebola para avaliação de toxicidade de efluentes industriais. MsC. Dissertation. Universidade de São Paulo. 81p. http://dx.doi.org/ 10.11606/D.6.1998.tde-08112013-121210

RIBEIRO, I.A. 1999. Teste de raízes de cebola para avaliação de toxicidade de efluentes industriais. Engenharia Sanitária e Ambiental 4(3)108-12.

ROSSETTI.V., FILIPPINI, M., SVERCEL, M., BARBOUR, A.D. \& BAGHERI, H.C. 2011. Emergent multicellular life cycles in filamentous bacteria owing to density-dependent population dynamics. J. R. Soc. Interface 8(65)1772. http://dx.doi. org/10.1098/rsif.2011.0102

STALEY, Z.R., HARWOOD, V.J. \& ROHR, J.R. 2015. Review Articles: A synthesis of the effects of pesticides on microbial persistence in aquatic ecosystems. Crit. Rev. Toxicol. 45(10)81336. http://dx.doi.org/10.3109/10408444.2015.1065471

STAUBER, J.L. \& DAVIES, C.M. 2000. Use and limitations of microbial bioassays for assessing copper bioavailability in the aquatic environment. Environ. Rev. 8(4):255-301. http://dx.doi. org/10.1139/a00-010

WANG , M., ZHENMEI, L. \& MIN, H. 2011. Responses of microorganisms to pollutants and its application in environmental risk assessment. In: Visser, J.E. (ed), Ecotoxicology around the globe. Nova Science Publishers, pp. 179-194.

WIDDER, S., ALLEN, R.J., PFEIFFER, T. \& CURTIS, T.P. 2016. Mini Review: Challenges in microbial ecology: building predictive understanding of community function and dynamics. ISME J. 10(11):2557-68. http://dx.doi.org/10.1038/ ismej.2016.45

TEELING, H., FUCHS, B.M., BECHER, D. \& KLOCKOW, C. 2012. Substrate-controlled succession of marine bacterioplankton populations induced by a phytoplanction bloom. Science 336(6081)608-11. http://dx.doi.org/10.1126/science.1218344

Thorpe, L. 2014. The Kant dictionary. Bloomsbury academic, 248p. 